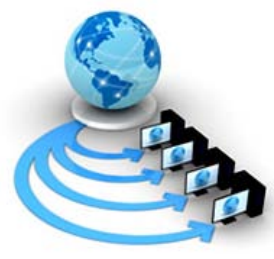

Volume 9, No. 2, March-April 2018

International Journal of Advanced Research in Computer Science

REVIEW ARTICLE

Available Online at www.ijarcs.info

\title{
LITERATURE REVIEW ON SECURITY OF IOT
}

\author{
Sombir \\ Student, CSE Department \\ UIET, M.D University \\ Rohtak, India
}

\author{
Kamna Solanki \\ Assistant Professor, CSE Department \\ UIET, M.D University \\ Rohtak, India
}

\begin{abstract}
This paper reflects light on the IoT and its security aspects. Internet of things is an evolving automation in the world. As numerous gadgets are mingled together to form an interconnection of a data hub. IoT automation is incrementing day by day as the devices connected to the internet are increasing time to time. This paper discussed the various security parameters in which IoT network system acts a malicious component. Many researchers discussed about the security prespective of it but did not find the analytical solution. It is one of the astonishing fact that with emerging technology there would be security aspects associated with it and can be taken care from time to time. No network system in the world is 100 percent accurate in the functioning field.
\end{abstract}

Keywords: IoT, components, applications, security measures

\section{INTRODUCTION}

Internet of things is an emerging automation associating all smart gadgets to the internet. Sensors or actuators are the parameters to transmit data across the web. Connecting everyday things installed with electronic software and sensors to the internet enabling them to collect and exchange data. For example for smart home by utilizing he IoT methodology here user can switch on or off his fan when he is not present at home. Sensor automation have a platform which can connect my car to a IoT tracking application to keep analyzing fuel meter and also the car GPS location to track the car in case of theft. This can be done as all gadgets are associated to same platform. So internet becomes the legitimate connection by which you connect all the component or things to a common automation. In the year 1999 IOT gets an identity. Kevin Asthon discovers the article "internet of things" and well-established MIT's Auto ID center, round the globe study area of network conncetion by academic testing area pinpoint on Radio Frequency Identification and the IoT. The IOT sometimes also referred as internet of everything (IOE). In this collected data is passed and act on everything and acquire the environments using installed sensors processing system and transmission hardware. It refers as "Smart" devices. This process termed as machine to machine (M2M). The approach of IoT rise some buzzing acceptance in the year 2010 because the data leaked of google street view service had not developed 360 degree view but also reserved tons of personal data of users in their data hub. As this malicious event occurred in coming year Chinese government will make IoT strategy as their priority. The market community Gartner discovered "Hype-cycle for immense automation" combines advanced approaches and emerging phenomena.

\section{Advantages of IoT}

\section{$>$ Efficient resource utilization}

Would you efficiently utilize the resource that are available if you have a smart data store hub. Which can interact with everything enough computational power if it has enough understanding of how things can work to each other. This resources could be in terms of monitoring it and could be in terms of natural resources it could also be an input taken up by the things as an input and so far. So all this can be more efficient if you have a platform. Which is smarter and interconnected as well.

\section{$>$ Human effort minimizing}

As part from it minimizing the human effort involved. This is same with everyone and that of the one of the major reasons why IOT is become popular today and the concept of smart homes is always growing as well in the same prospective. Because if the system itself is able to do most of work at home then need to put in much effort and relax at home with my having to worry about anything.

\section{$>$ Save time}

If the resource are utilize are more efficiently. Then again is going to save lot more time as well. All in all any benefit of IOT will in turn to help save lot of time as well.

\section{$>$ Development of AI through IoT}

Today most of us have had at a level of interaction with an artificial intelligence or virtual intelligences as well. This could be a personal assist like SIRI and it could be assist application like google assist. Now if I have system where all this component in turn and things are interconnected then all the security presenter each of things is going to get multiplied is going to much build is much more secure system. 
$>$ Improved security

A part from that level of security would be integrity to the platforms and itself is going to be quit use. So the overall security with respect to everything is going to be increase multiple times.

\section{IoT Features}

Any technology that is available today has not reached hundred percent capability and it always has chance to extend. IoT is one of the major technology in the world today. That can help any other technology reach true and complete potential.

Connect - Here basically what you need to work on is need to ensure that there is a connectivity all the things around all the necessary things to the IoT platform.

1. Device virtualization - Standardize integration of devices.

2. Huge speed forecast : - Empower decisive, guarded and bi-directional.

3. Endpoint management: - Manage device end point identity.

Analyze - Now analyze can around me there each are going to generate some amount of data. Now this data needs to be collected and it needs to be analyze to build a business intelligence solution. If I have a good inside from the data that is gather from all of this. Then definitely call my assistant as a smart system.

1. Stream processing: - live data analytics of approaching information with event association, selecting and interrelation.

2. Data enhancement - information regarding concepts and develop complex streams.

3. Event reserve area - Problem and resolve huge amount of information which is associated with cloud storage and enable big data analytic advancement.

Integration within system : - Finally what happen in order to improvise and improve your system need to associate with various model to user experience.

Let say this a modification model that is there or let going to be directly connect to my providers and this providers called to be amazon, flipkart and could also be my retail store that is next to be.

Let say its act of milk retail store what could be notification and he would be send milk to me correct destination and some things let can be done.

Let say my coffee machine require special order and this order is almost complete then what could my system go online to amazon and purchase these order for me. So there are lot of things can do integrate this various model and improve the overall experience of a single user or a group of users.
1. Enterprise connection - effectively expedition crucial IoT and its associated events in the network system.

2. Regulation authority and authority: - pass message to gadget from wireless means of network for example mobile technology in the emerging technology.

\section{APPLICATIONS OF IOT}

\section{Medical and health care system}

A very useful Application are medical and health care system. Because the lack of medical staffs, facility, treatment level low, travelling system, etc. this is mainly in rural areas. This is not connected to the IOT network. It is also utilized the representation of disease prevention and detection capability to research the address with the help of Remote Monitoring and Management Platform of Healthcare Information (RMMO-HI). To reach the address prevention the disease. It can also be used the human body sensor that means this sensor loaded in human body and know all about of them. Healthcare can be utilized for making team as to track the members of the staff whether they are present or not for example their attendance and patients will track by which medicines they are being diagnosed. Real time monitoring activity and personal data can give a device on tired an exercise provide early morning signal for critical illness to improve track delivery. This same technology can also keep us helping by helping to deliver to medical services every one affordable cast. The pro smart system to connect many devices to the IOT. This can be increase the amount of data and stored of data tom creating need for more powerful infrastructure.

\section{Smart homes}

At this time smart homes technology are used in all over world. As its all items are used in yours homes are connected to the internet. Like the electronic items are washing machine, refrigerator, oven etc. Also connected item in your home to the internet are power, telecommunication, gas, water, doors, lighting, air conditioning, these item are controlled by the remote. Some other item are controlled by remote are radio, television, home security, and healthcare. Our technology allow management of lights heating and any home appliances control the smart system and when connected to the cloud enables to remote monitoring and control are home. HD solutions deliver the music system and moves to information all the connected devices. They need when home entertainment video transcoding, stunning graphics for live action gaming and action in home connectivity.

\section{Smart Environmental}

Smart environment means that how can the human kind be advanced and helped by utilizing proper resources at best suited time. The smart environment refers such as temperature, humidity in the air which is breathe by the living beings by the utilization of the sensors which can 
easily sense the environment. Mainly this application purposes is every sensor attach to a device in the IOT environment has to write a program code for data collection and this program is complicated and burdensome. This problem solved make a new method these are smart sensor interface in IOT environment. High efficiency motor drivers (HD) technology also have protect the environment by reducing the fuel consumption and co emission and HD infotainment and navigation in car control and increase the enjoyment overall travel experience.

\section{4. $\quad$ Asset tracking}

Companies moving large quantities of goods are refer to IOT by putting sensor on product or contains to keep track of goods on the move. This can be used the product making is sire not to minimal. Using the smart devices provide the accurate measurement and goods are very well to all company or it will be moved to real time. Mainly three example of industry are retail, manufacturing and agriculture IOT asset tracking to their benefit.

\section{RELATED WORK}

Wang et.al. "Research on Security Management for Internet of Things"

The authors have discussed security management of IoT. In this paper, the authors have explained machine to machine communication using attributes of objects which are coined as Static or Dynamic. Static directly stored in the label on the other hand Dynamic are detecting the sensor. IoT is attached to machine which in turn to the internet and the heterogeneous network integration is the trend of network development. Network resource management respectively work for different algorithm load balance, power control, congestion control, control switch etc. The main aim of this paper is M2M transmission, sensor and security [1].

\begin{abstract}
Alam et.al. "Interoperability of Security-Enabled Internet of Things"

The authors have proposed layered architecture of the IoT framework. It will be focused on security reasoning interoperability aspects and also the crucial challenges. Internet of things is based on the specific application and context. In internet of things architecture each layer is responsible for separate concern [2].
\end{abstract}

Suo et.al. "Security in the Internet of Things: A Review" The authors have discussed subsequent application associated with the industry. In this paper, the authors have proposed the encryption technique, network security, shielding sensor information [3].

\section{Chen "AN IBE-BASED SECURITY SCHEME ON INTERNET OF THINGS”}

The authors have discussed IBE- identity based encryption also be used by men are layer system. Used three layers are applied middleware, and perceptron layer. It also used of RFID-radio frequency identification. This is the contactless recognition system. In this paper, the authors discussed the elliptic curve cryptosystem and identity based encryption. Both of them are used the public key cryptography [4].
Poslad et.al. "International Workshop on Adaptive Security \& Privacy management for the Internet of Things"

The authors have discussed brief of the IoT, its security and security threats associated to it. In this paper, the authors discussed many types of security threats are discussed in this paper like these are unattended devices low power device, user managed device and many security threats are discussed this paper [5].

Razzaq et.al. "Security Issues in the Internet of Things (IoT): A Comprehensive Study"

The authors have discussed how many people connect via internet in the world. In future, then it will be changing the scene of life and all activity in your daily life. In case of IOT devices, security is $70 \%$ devices are attacked. It also discusses the security concept like industry, medical devices, smart homes, and many concepts in the IOT. In this paper, the authors discussed secure network to shield form attack authentic devices and access control means only authorized person can utilize it [6].

\section{Raja et.al. “AN INTERNET OF THINGS (IOT) BASED SECURITY ALERT SYSTEM USING RASPBERRY PI”}

The authors have discussed new security concepts are coming on the market. Sensory play an important role in security system. Wireless security is more popular at this time because it says easy installation and simple nature. Its main work is in the home security system. The main drawback is not easy to implement its expensive and complex. . In this paper, the authors discussed the RASPBERRY PI used in python script and typed the code to run the Raspbion OS. It provides the E-mail and SMS system and also work the motion detection [7].

Tahir et.al. "Internet of Things (IoT): An Overview of Applications and Security Issues Regarding Implementation"

The authors have discussed utilization of the internet and mobile communication. It implies connect the Wi-fi, Bluetooth and many other gadgets. . In this paper, the authors discussed the replay attack [8].

Barcena et.al. "Insecurity in the Internet of Things"

The authors have discussed various security allotment in different applications like home gadgets, Physical access and the Ethernet. Home devices provide the network and how can it devices are used. In case of attack can provide the physical access. It also used the malware, malicious software, it is able to provide interaction with the smart homes [9].

\section{Kumar et.al. "A Survey on Internet of Things: Security} and Privacy Issues"

The authors have discussed people are connected to the internet in any place and any time with the help of sensing devices and network. This is also the same work for other researchers like to define the architecture and application of the IOT. But one is differently used are MAC (Medium Access Control). It also more work is pending because the process of IOT security can never be ended [10]. 
Suchitra and Vandana "Internet of Things and Security Issues"

The authors have discussed the various security requirement and challenges in the IOT and also discuss the various researchers can define this topic in the past. . In this paper, the authors discussed DOS (Denial of Service) are utilized and also explains the DOS attack [11].

Kai et.al. "Security and privacy mechanism for health internet of things"

The authors have discussed smart health in the IOT. It is very different, think in the case of security in healthcare. It mainly works on the IOT is the Information and Communication Technology (ICT) industry. In this paper, the authors discussed security of health care system. Its follows the new strategy in the health of the IOT [12].

\section{Zhao et.al. "A Survey on the Internet of Things Security"}

The authors have discussed IoT, its application and usage in the future. In this paper, the authors proposed to find the solution to minimize the threat of IoT [13].

\section{CONCLUSION}

As with the emerging world and technology at every second the number of gadgets mingled with the internet connectivity is incrementing. IoT provides us a manual labor effortless system. Despite the fact it has limitation too like the security and privacy measure. As devices are increasing into a network therefore it will be a difficult task for the operating system to manage all these new comers devices at a efficient rate of mechanism so security is a very serious issue as users are very serious about their personal data leak, loss of information and few more. As we know internet is prone to attacks which may change malfunctioning of the normal system network. Many researchers are working on different aspects like the data prevention, how to make more health systems, how to establish more intelligence agriculture system etc.

\section{REFERENCES}

Kun Wang Jianming Bao and Meng Wu Weifeng $\mathrm{Lu}$ "Research on Security Management for Internet of Things” 2010 International Conference on Computer Application and System Modeling Vol-I5 pg- 133 to 137.

[2] Sarfraz Alam, Mohammad M. R. Chowdhury and Josef Noll "Interoperability of Security-Enabled IoT” Wireless Commun (2011) vol-61:567-586 .
[3] Hui Suo, Jiafu Wan, Caifeng Zou and Jianqi Liu "Security in the Internet of Things: A Review" 2012 International Conference on Computer Science and Electronics Engineering pg- 648 to 651.

[4] Wang Chen "AN IBE-BASED SECURITY SCHEME ON INTERNET OF THING 2012 Proceedings of IEEE CCIS pg- 1046 to 1049.

[5] Stefan Poslad, Mohamed Hamdi and Habtamu Abie” International Workshop on Adaptive Security \& Privacy management for the Internet of Things” 2013 pg-373 to 378

[6] Mirza Abdur Razzaq, Muhammad Ali Qureshi, Sajid Habib Gill, Saleem Ullah "Security Issues in the Internet of Things (IoT): A Comprehensive Study” International Journal of Advanced Computer Science and Applications, Vol. 8, No. 6, 2017.

[7] A.Arun Raja, R.Naveedha, G.Niranjanadevi and V.Roobini “AN INTERNET OF THINGS (IOT) BASED SECURITY ALERT SYSTEM USING RASPBERRY PI” ASIA PACIFIC INTERNATIONAL JOURNAL OF ENGINEERING SCIENCE Vol. 02 (01) (2016) 37-41

[8] Hafsa Tahir, Ayesha Kanwer and M. Junaid "Internet of Things (IoT): An Overview of Applications and Security Issues Regarding Implementation” INTERNATIONAL JOURNAL OF MULTIDISCIPLINARY SCIENCES AND ENGINEERING, VOL. 7, NO. 1, JANUARY 2016.

[9] Mario Ballano Barcena and Candid Wueest "Insecurity in the Internet of Things” http:// www.symantec.com Version 1.0 - March 12, 2015.

[10] J. Sathish Kumar and Dhiren R. Patel "A Survey on Internet of Things: Security and Privacy Issues" International Journal of Computer Applications (0975 8887) Volume 90 - No 11, March 2014 pg-20 to 26.

[11] Suchitra.C and Vandana C.P "Internet of Things and Security Issues” IJCSMC, Vol. 5, Issue. 1, January 2016, pg.133 - 139 .

[12] KANG Kai1, PANG Zhi-bo and WANG Cong "Security and privacy mechanism for health internet of thing" December 2013, 20(Suppl. 2): pg-64-68 www.sciencedirect.com/science/journal/10058885.

[13] Kai Zhao and Lina Ge "Survey on the Internet of Things Security” 2013 Ninth International Conference on Computational Intelligence and Security. pg-663 to 667. 